2013-09

\title{
Visual and linguistic cues to graspable
} objects

\section{Myachykov, A}

http://hdl.handle.net/10026.1/3603

10.1007/s00221-013-3616-z

Experimental Brain Research

Springer Science and Business Media LLC

All content in PEARL is protected by copyright law. Author manuscripts are made available in accordance with publisher policies. Please cite only the published version using the details provided on the item record or document. In the absence of an open licence (e.g. Creative Commons), permissions for further reuse of content should be sought from the publisher or author. 
PREPRINT - Myachykov A., Ellis R., Cangelosi A., Fischer M.H. (2013). Visual and linguistic cues to graspable objects and graspable parts. Experimental Brain Research, 229(4), 545-559. DOI: 10.1007/s00221-013-3616-z

Visual and linguistic cues to graspable objects and graspable parts.

Andriy Myachykov, Rob Ellis, Angelo Cangelosi, and Martin. H. Fischer

\section{Author note}

Andriy Myachykov, Department of Psychology, Northumbria University, Newcastle upon Tyne.

Rob Ellis and Angelo Cangelosi, School of Psychology, University of Plymouth.

Martin H. Fischer, School of Psychology, University of Potsdam.

This research was supported by the EPSRC grant EP/F026471.

Correspondence concerning this article should be addressed to Andriy Myachykov, Department of Psychology, Northumbria University, Northumberland Building, Newcastle upon Tyne, NE1 8ST, United Kingdom, Tel.: +44-191-227-31-58, Fax: +44-191-227-4515, e-mail: andriy.myachykov@northumbria.ac.uk

Word count: 7575 words. 


\begin{abstract}
Mentally representing manipulable objects involves automatic encoding of their corresponding affordances - options for interacting with the object. Two experiments investigated how activation of objects' manual affordances is triggered by visual and linguistic cues and whether graspable object parts play a special role in this process. First, analysis of participants' motor and oculomotor behaviour confirmed that perceptual and linguistic cues potentiate activation of grasp affordances. Second, a differential visualattention mechanism is proposed for the activation of individual compatibility effects associated with target and distractor objects. Third, we registered an implicit attention attraction effect from an object's handles suggesting that graspable parts automatically attract attention during object identification. Fourth, this effect was further amplified by visual but not linguistic cueing manipulations. The latter finding confirms a recent hypothesis about differential roles of visual and linguistic information about perceived objects and the resulting action planning processes. Our results inform current theories of vision for action.
\end{abstract}

152 words.

Key words: grasp affordances, naming, visual attention, object recognition. 
Visual and Linguistic Cues to Manual Grasp Affordances

Interacting with real world and man-made objects is an everyday routine task.

Consider the following example. Your friend asks you to pass a cup that is on the table in front of you. After hearing the word "cup", you need to visually locate it, reach for it, seize it by its body or handle, pass it to your friend, and, finally, release the grip. This is a simple and effortless task; yet it requires well-orchestrated contributions from different perceptual, linguistic, and motor circuits of the brain that control the performance of the corresponding sensors and effectors. According to vision-for-action (e.g., Goodale, 2011, for a recent review) or mental simulation (e.g., Barsalou, 2008) theories, we are extremely efficient in these routine tasks because simply hearing a word related to a graspable object or seeing the object itself, even without an intent to use it, activates sensorimotor areas of the brain associated with the actual potential object manipulation (Martin, 2008). The specific focus of this paper is on grasp affordances - the object's features associated with how we typically interact with graspable objects, for example, the cup in our previous example (Gibson, 1979; Michaels, 1989; 1993).

Experimental findings documenting compatibility effects (Tucker \& Ellis, 1998, 2001; Ellis \& Tucker, 2000; Ellis, Tucker, Symes, \& Vainio, 2007; Fischer \& Dahl, 2007) provide good empirical support to the idea that simply perceiving a graspable object activates associated grasp affordances. For example, Tucker and Ellis (1998) demonstrated that when classifying whether a graspable object was up-side or not, responses were easier (faster and more accurate) when the responding hand corresponded to the hand that would be optimal for grasping the depicted object. In contrast, when having to ignore an object in order to respond to the properties of another target object, responses were slower and less accurate when the optimal grasp associated with the ignored object was the same as that required as the response to the target (Ellis et al., 2007). These and similar findings suggest 
that simply seeing a graspable object activates the corresponding manual actions associated with it and that ignoring an object entails inhibiting these actions.

Retrieval of linguistic information about a graspable object also has consequences for affordance processing, such that preparing for a grasp may influence understanding of the words associated with similarly grasped objects, and hearing grasp-related words may facilitate visual processing of the objects. For example, Klatzky and colleagues (1989) verbally instructed a hand shape (e.g., pinch or clench). Once participants adopted the hand shape, they had to decide whether a particular action description (e.g., eat a carrot) was sensible or not. Adopting hand shapes that were congruent with the object referred to in the action description facilitated the sensibility decisions. This finding was confirmed and extended in more recent studies. Tucker and Ellis (2004), for example, demonstrated that showing a graspable object's name produced a congruency effect on responses similar to the one commonly registered in visual object categorization studies. Similar effects were reported in Bub, Masson, and Cree (2008) for both functional (grasping) and volumetric (lifting) actions (see also Lindemann, et al., 2006). Furthermore, studies by Masson, Bub, and Newton-Taylor (2008), Masson, Bub, and Warren (2008), and Bub and Masson (2010) found that the linguistic elicitation of affordances is not limited to single-word processing. In their studies, functional gestures were produced faster after participants had read or listened to sentences referring to objects that afford similar grasps.

Finally, a series of recent reports using a variant of the sentence-picture verification task showed that both functional (grasp) and manipulation (drink) verbs related to graspable objects give rise to affordance effects in a fashion similar to nouns (Ambrosini, et al., 2012; Borghi and Riggio, 2009; Constantini, et al., 2011). In a typical setup, (Borghi \& Riggio, 2009), participants first read imperative sentences (e.g., “Verb” at the nail.) with the verbs functionally related (e.g., grasp) or unrelated (e.g., look) to the object's affordance. The noun in the sentence was always the name of the target visual object 
displayed afterwards, and the objects were either power- or precision-grip affording. The task was to decide whether the object mentioned in the prime sentence was the same as the one they saw afterwards. Decision times were faster when the verbs in prime sentences were functional than when they were unrelated. Therefore, the verbs' functional semantics elicited an affordance effect similar to the effect of the object's names documented in earlier studies. Put together, the studies reviewed thus far demonstrate that both seeing a graspable object and hearing its name or a verb related to manipulating the object leads to sensorimotor simulation of the associated grasp affordances.

Typical manual manipulations with graspable objects are highly automatic and seemingly effortless. Therefore, first important question is whether the affordance effects are automatic or whether the viewer needs to pay specific attention to the perceived object or its graspable part (e.g., handle) in order for the affordance effect to emerge (Humphreys et al., 2010, for a recent review). A number of studies (e.g., di Pellegrino, Rafal, \& Tipper, 2005; Pappas \& Mack, 2008; Phillips \& Ward, 2002; Riggio, et al., 2008; Vainio, Ellis, \& Tucker, 2007) found that, regardless of whether the whole object or any of its parts is currently in the viewer's attentional focus, affordance effects are automatically evoked. Pappas and Mack (2008), for example, found that the affordance effect can be triggered outside of the viewer's conscious perception by co-present but undetected objects with similar affordances. Furthermore, Philips and Ward (2002) demonstrated that the graspable object's orientation facilitates corresponding hand responses in situations when the object itself is totally irrelevant to the task. Finally, Derbyshire et al. (2006) demonstrated the presence of affordance effects after an object was removed from the observer's view. Based on these and similar findings, Based on this evidence, one might conclude that object-directed attention is not a prerequisite for the emergence of the affordance effects.

Other reports, however, suggest that affordance effects may be attributed to the attentional shift induced by perceiving asymmetrical targets. Anderson, Yamagishi, and 
Karavia (2002) presented participants with both affording and nonaffording objects (i.e., with or without handles) in their canonical (horizontal) and noncanonical (slightly tilted clock-wise or counter-clockwise) orientation. Participants judged the direction of orientation change by making left-hand or right-hand responses. Importantly, the pattern of results did not change for affording and nonaffording objects, indicating that the object's conspicuous feature but not the orientation of its graspable part was responsible for the facilitation of the lateralized response. Finally, and in conflict with the notion of strictly automatic activation of affordances, attention to the semantic properties (e.g., goal-directed use) of the graspable objects reliably modulates the affordance effect: When the experimental task is relevant to the grasp-related potential of the perceived object, the resulting affordance effect seems to be stronger (Creem \& Proffitt, 2001; Shuch et al., 2010; Tipper, Paul, \& Hayes, 2006). In addition, data on grasp-aperture priming clearly points to the existence of a top-down link between grasping and attention in that preparing to manipulate objects modulates attentional control. More specifically, preparing the hand for appropriate grasping action affects the response to graspable objects (Craighero et al., 1999) and hands (Craighero et al., 2002). Recently, Tschentscher and Fischer (2008) found that combining directional and aperture cues led to the registration of aperture congruency effects for power grips. Adamo and Ferber (2009) demonstrated that presentation of manipulable tools (or their names) together with the objects these tools are usually used upon results in an action priming effect. Similarly, Symes et al. (2008) showed that grasp preparation improved participants' perception of grasp-congruent objects. This motor attention mechanism selectively inhibited competing motor programs associated with the same object that could potentiate erroneous actions (Loach et al., 2008). A recent report by Symes and colleagues (2010) suggested that the initial attentional state of the observer matters: Top-down motor attention induced by grasp preparation improved visual detection of grasp-congruent targets only when the latter were not visually salient. 
In summary, despite this wide range of findings linking affordances and attention, the exact nature of the interaction between bottom-up and top-down attentional control of the affordance effect is still unclear. One recent theoretical proposal suggests that the exact dependence of affordance effects on the allocation of attention varies between stable and variable types of affordances (Borghi \& Riggio, 2009). Stable (stored or core) affordances are intrinsic properties of the affording objects; such as, general graspability, size, or weight. These are hypothesized to be stored permanently as components of the object's off-line representation, elicited automatically (e.g., without special-attention prerequisites) and independent of specific visual context (e.g., spatial orientation). Variable (or situated) affordances, on the other hand, are emergent and context-specific object properties. As such, they are not stored permanently, subject to the object's orientation, and short-lived. Due to their emergent properties, variable affordances should require specific attention on the object or its graspable parts. To better understand this distinction think of a cup. Its stable affordances will result from its general graspability, both by means of wrapping one's hand around the cup's body and by using its canonical grasp-related part, i.e., the handle. This general graspability of a cup is expected to be encoded in its stored (or offline) representation independent of how the cup is spatially presented.

Further developing the proposed distinction, Borghi (2012) makes a series of valuable predictions with regard to the differential effects of linguistic (e.g., names) and visual cues to affordance effects. Because only stable affordances are expected to be stored permanently, top-down linguistic cues should only activate this type of affordance as it would be highly uneconomical to permanently store all possible orientations a cup can have. Bottom-up visual cues, on the other hand, should facilitate the extraction of variable affordances, such as well-documented compatibility (e.g., handle-orientation) effects. Compatibility effects resulting from handle orientation (leftward/rightward), in turn, reflect the cup's variable affordances as they will have to emerge within a given visual context. 
Because of their emergent nature, variable affordance effects should be dependent on visually directed attention and independent of linguistic cues.

The specified distinction between stable and variable affordances becomes crucially important for the predictions for the studies reported below. Importantly, in both studies we do not only use manual-response reaction time data as the affordance-specific evidence; we also analyse participants' oculomotor behaviour in order to further investigate the time-course and the early functional dynamics of the emergence of the affordance effects. In Experiment 1, we examine the availability of a target's affordances depending on its attentional status and the presence of another object with similar or opposite affordances. Experiment 2 replicates and extends this approach by adding linguistic cues. The presentation of the data from both studies is followed by general discussion about the nature of the distinct roles of and the interplay between, the visual and the linguistic processing domains in their ability to evoke manual affordance effects.

\section{Experiment 1}

The main goal of Experiment 1 was to investigate how the affordance effect in twoobject displays depends on the saliency of the target (being either visually unfocused or visually focused; Symes et al., 2001), on the orientation of the distractor (response inhibition; Ellis et al., 2007) and on attention to the target's graspable part. The last issue was addressed by recording eye movements as participants decided with lateralized buttons whether the target was a kitchen utensil or a tool.

\section{Method}

\section{Design}

Our three main questions and the corresponding analyses motivated our experimental design. For the purposes of evaluating the effects of visual focus and distractor orientation, three factors were independently manipulated: Target Congruency (Congruent/Incongruent), Distractor Congruency (Congruent/Incongruent), and Visual 
Focus (Target/Distractor). This resulted in a $2 \times 2 \times 2$ within-subjects design. The identity of the target object was indicated to participants by means of changing its colour from natural to green. A target or a distractor was congruent when the direction of its handle (left/right) was concordant with the response hand (left/right). In half of the trials, the object in the visually focused position became the target; in the other half it was the distractor (see details of Visual Focus manipulation below).

Following Ellis et al. (2007) we expected opposite effects from the responsecongruent targets and the response-congruent distractors on manual-response reaction times (RT): Congruent targets should facilitate behavioural performance while congruent distractors should inhibit it. The Visual Focus manipulation should make one of the two objects more accessible for processing than the other, allowing us to test for possible attentional enhancement of both the target-bound and the distractor-bound congruency effects. The general hypothesis is that the more salient of the two co-presented objects would be processed faster, therefore enhancing the congruency effects from this salient object (Symes et al., 2010). This should result in the registration of interactions between Visual Focus and the two congruency effects.

For an evaluation of the role of the graspable object part we concentrated on the viewers' oculomotor behaviour specifically related to the activation of the affordance effects. As a result, we modelled an object-based rather than target-distractor based analysis with the data coded according to the following factors: Visual Focus (Focused/Unfocused), Object Status (Target/Distractor), and Object Part (Body/Handle). This design is crucial for the analysis of how visual interrogation of objects' manipulable parts (e.g., handles) changed when participants prepared and executed target responses in the presence of a distractor. The main hypothesis for this analysis was that viewers should attend proportionally more to manipulable parts of the objects (e.g., handles) than on their bodies. 
One specific temporal interest period was used for both analyses. The interest period started with one of the two displayed objects turning green (target onset) and ended with the manual response (key press) to indicate the target's identity. The following manual and oculomotor behavioural measurements were treated as dependent factors: (1) manual response latencies to identify the target and (2) proportional Total Dwell Times (accumulated fixation durations divided by area in pixels, expressed as ms per interest area or MIA) on the objects and their graspable parts (i.e., handles).

\section{Materials}

Experimental materials consisted of 256 colour photographic displays portraying two manipulable objects - one was always a mechanical tool and the other was always a kitchen utensil (see example in Figure 1).

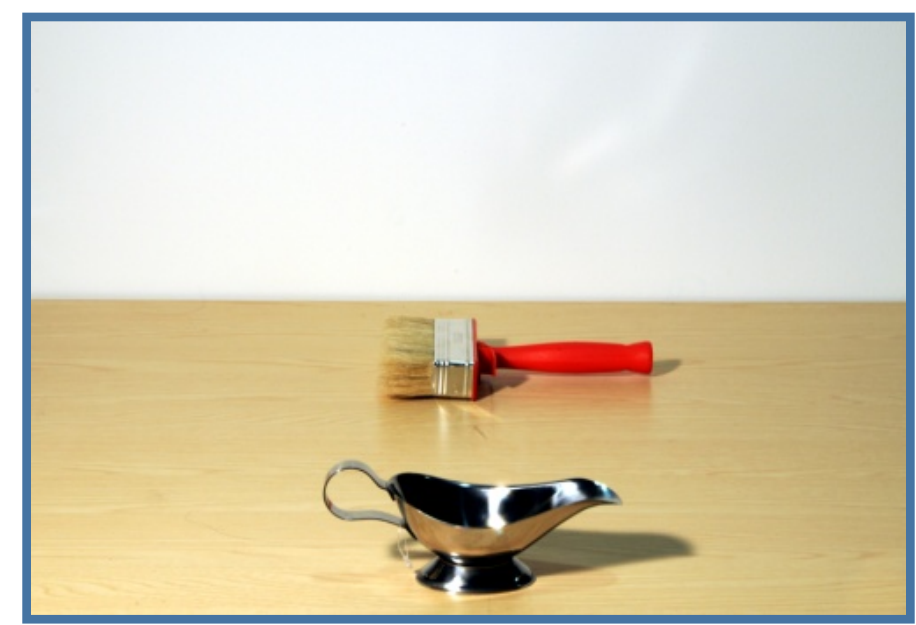

Figure 1. Example of experimental materials.

All experimental materials were in landscape format with horizontal-to-vertical size dimensions of $1024 \times 768$ pixels. The "kitchen utensils" were cup, pan, saucer, and strainer. The "tools" were brush, chisel, saw, and screwdriver. We implemented all 16 possible tool-utensil combinations counterbalancing their presentation across the experimental conditions. The photographed objects were positioned on a flat surface against a light background. The objects were photographed so that one appeared farther from the viewer while the other appeared closer (see Figure 1). Both objects were 
presented with their bodies and handles perfectly visible. The objects were presented with their handles parallel to each other and always pointing either to the right or to the left side of the screen. Experimental materials were not controlled for size, luminance-related properties, or familiarity to the participants. A random selection of 16 experimental trials ( 2 per experimental condition) was used in the practice session.

For the purposes of our eye-tracking analysis, we used SR Research Experiment Builder $^{\odot}$ to create original and mirror free-hand body-specific and handle-specific interest area sets for each of the objects used in the study. We implemented these interest area sets in each of the 256 pictures used in our studies (see example in Figure 2).

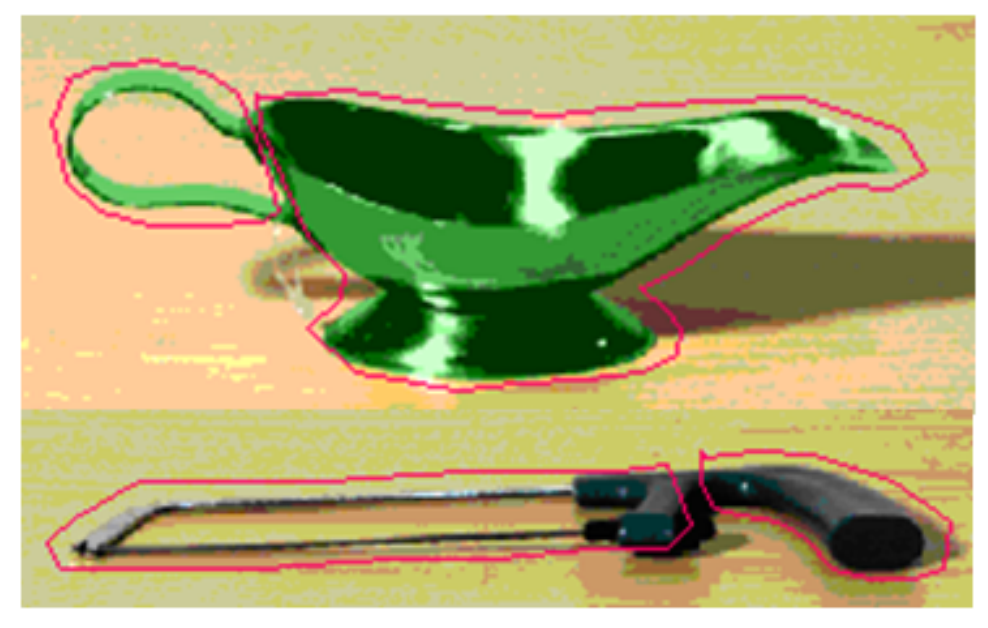

Figure 2. Body-specific and handle-specific interest areas.

\section{Apparatus}

The experiment was implemented in SR-Research Experiment Builder ${ }^{\odot}$ Version 1.5.201. An EyeLink ${ }^{\odot} 1000$ desk-mounted eye tracker with $1000 \mathrm{~Hz}$ sampling rate monitored participants' eye movements. The materials were presented on a 19' ViewSonic $^{\odot}$ G90fB monitor of a DELL ${ }^{\odot}$ Optiplex 755 desktop computer running at a display refresh rate of $90 \mathrm{~Hz}$. A chin rest restricted head movements. Manual response latencies were recorded time-locked to eye-tracking data with the help of the Microsoft ${ }^{\odot}$ Sidewinder game-pad integrated with the EyeLink ${ }^{\mathcal{O}}$ eye-tracking system. The eye-tracking data were extracted and filtered using SR-Research Data Viewer ${ }^{\odot}$ Version 1.91. 
Participants' handedness was assessed with a modified version of Annett's handedness questionnaire (Annett, 1967; 1970).

\section{Participants}

Twenty one native speakers of English (4 males) studying at Dundee University in Scotland participated for course credit or $£ 6$. Their mean age was 19.7 years and all had normal or corrected-to-normal vision. Their average handedness score was 34.3 (range 2936), indicating they were all right-handed.

\section{Procedure}

Before each session, the experimenter collected the participant's written consent and administered the handedness questionnaire. Then the participant was positioned approximately $60 \mathrm{~cm}$ in front of the monitor. Viewing was binocular, but only the dominant eye was tracked (Roth, 1992). Before the main experiment each participant saw a randomized sequence of 16 practice trials -2 from each of the experimental conditions. After that, the eye-tracking equipment was calibrated.

During the experimental session, each participant was presented with an individually randomized sequence of 256 trials, such that a maximum of two trials from the same experimental condition were presented in succession. A typical trial is portrayed in Figure 3.

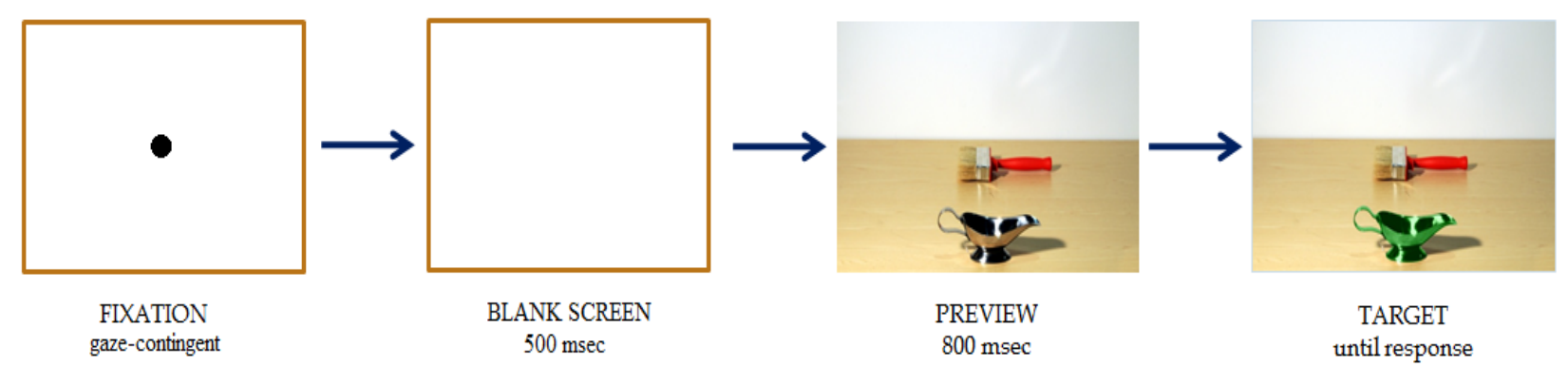

Figure 3. Experimental trial sequence in Experiment 1.

Each experimental trial started with the presentation of black central fixation dot (radius 20 pixels). The spatial position of the fixation dot always corresponded with the 
location of the backgrounded object in the subsequently presented target display putting this backgrounded object in the focus of visual attention when the preview display appeared on the screen. The participant had to fixate the dot for a minimum of $150 \mathrm{msec}$. before it was replaced by a $500 \mathrm{msec}$. blank screen, followed by the $800 \mathrm{msec}$. preview display. After $800 \mathrm{msec}$. preview, one of the objects would change its natural colour to green. This change signalled to the participant the identity of the target. Once that happened, the participant had to indicate whether this target was a utensil or a tool by pressing either the left or the right key on the game pad, according to the experimental instruction. The resulting manual reaction time (RT) was the time interval between the onset of the target (colour changing event) and the time the participant pressed the response key.

Participants were randomly assigned to one of the two instruction groups. Group 1 pressed the left key if the target was a kitchen utensil and the right key if it was a tool. Participants in Group 2 received the opposite response rule. Participants were told to look at the central fixation point at the beginning of each trial, to await the next display, to freely explore the preview display, and to press the correct response key as soon as they noticed the change of color. Each participant was debriefed after data collection to establish that the purposes of the study had remained unknown.

\section{Results}

Data from 2 participants were excluded due to high error rate (over 15\%) and data from one more participant were excluded due to inflated RTs (over 2 standard deviations above the group mean). Analyses were performed on the data from the remaining 18 participants unless discussed separately. As accuracy was high (98\%) no error analyses were performed. There was no effect of Instruction Group on any dependent variables; therefore, we aggregated across this factor. 
We eliminated trials with incorrect responses or RTs outside $240-1300 \mathrm{~ms}$, as well as trials with RTs outside of 2 standard-deviations around an individual participant's mean. This left us with $90 \%$ of the data available for statistical analysis.

Average manual RT in Experiment 1 was $592 \mathrm{~ms}$. A 2x2x2 analysis of variance (ANOVA) revealed a marginally reliable effect of Visual Focus $(F(1,17)=3.391, p=$ .083 ) with focused (i.e., backgrounded) targets processed $9 \mathrm{~ms}$ faster ( $M s=588$ and 597 ms, respectively) than unfocused (i.e., foregrounded) targets. This initial finding confirms our general expectation that the central fixation dot effectively served as a visual cue to the subsequently presented background object's location (Posner, 1980). These visually focused objects, therefore, were preferentially attended and processed faster. Both manual RT and eye-tracking data analyses in both experiments consistently and repeatedly confirm this initial finding (see below).

Another marginally reliable effect was the effect of Distractor Congruency $(F(1,17)$ $=3.567, p=.076)$ : RTs to identify targets were $7 \mathrm{~ms}$ slower when the accompanying distractors were response-congruent $(M=596 \mathrm{~ms})$ than when they were responseincongruent $(M=589 \mathrm{~ms})$. This trend replicates the inhibitory effect from congruent distractors reported before (e.g., Ellis, et al., 2007). Although our analysis failed to register an individual effect of Target Congruency $(F(1,17)=.059, \mathrm{p}>.05)$, it revealed a reliable interaction between Visual Focus and Target Congruency $(F(1,17)=6.489, p<.05)($ see Figure 4). 


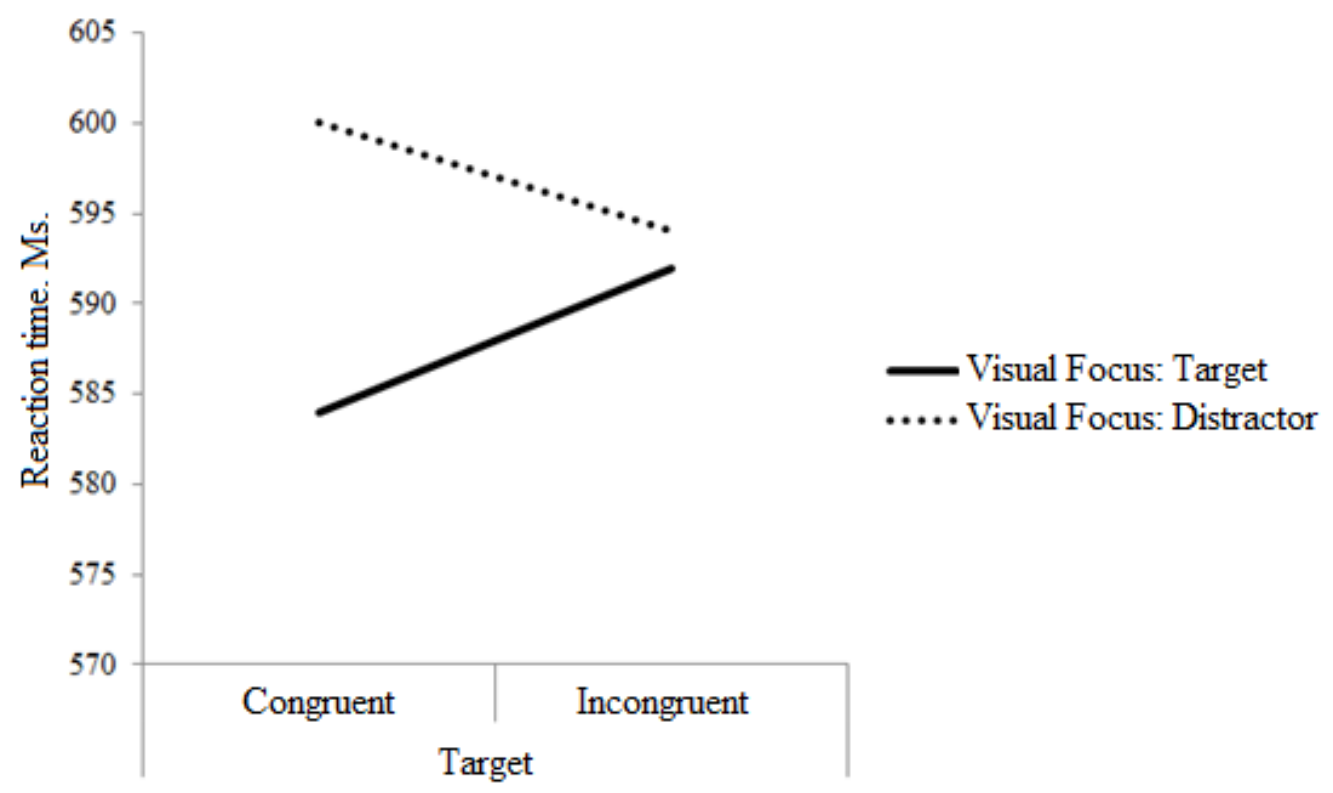

Figure

4. Experiment 1. Manual RT data for Target detection. Target Position x Target

Congruency.

The strongest combined facilitation from the two factors was observed when the visually focused targets were presented as response-congruent. A more detailed examination of this interaction by means of pair-wise t-tests revealed that participants were $17 \mathrm{~ms}$ faster to detect the identity of congruent targets in the visually focused position $(M=$ $584 \mathrm{~ms})$ than when the distractor was in focus $(M=601 \mathrm{~ms}), t(17)=-2.998, p=.008$. This confirms our post-hoc interpretation of the direction of the target position effect (see above): Given the design of our experimental materials, presenting an object in the visual focus corresponded with the location of the previously established attentional focus. This resulted in a faster recognition of the visually focused objects via attentional facilitation. Observed interaction pattern partially replicates previous findings by registering the significant difference in absolute RTs between the visually focused and the visually unfocused objects in case of congruency. It also reveals a positive compatibility effect for the visually focused targets and a negative compatibility effect for the visually focused 
distractors. The latter finding demonstrates that spatially guided visual attention gates target object recognition: Everything outside it is inhibited including the associated actions.

\section{Eye-tracking analysis}

In our eye-tracking analysis we were asking the two following general questions: (1) Do graspable parts implicitly attract special attention when people view and identify graspable objects and (2) does visual focusing amplify this potential effect. So, we implemented a 3way model with the following factors: Visual Focus (Focused/Unfocused) X Object Status (Target/Distractor) X Object Part (Body/Handle). ANOVA on proportional Total Dwell Times (accumulated fixation durations divided by area in pixels, expressed as ms per interest area or MIA) confirmed reliable main effects of all three independent factors: Visual Focus $(F(1$, a 16$)=75.508, p<.001)$, Object Status $(F(1,16)=27.731, p<.001)$, and Object Part $(F(1,16)=127.755, p<.001)$. Participants looked more at objects in focus than at those that were outside of focus ( $M s=9$ MIA and 3 MIA, respectively). Participants also looked more at targets than distractors ( $M s=7$ MIA and 6 MIA, respectively). Most importantly, participants looked proportionally longer at handles $(M=7 \mathrm{MIA})$ than at bodies ( $M=5$ MIA). Interaction between Visual Focus (focused/unfocused) and Object Part (body/handle) was also reliable, $F(1,16)=39.083, p<.001$ (see Figure 5).

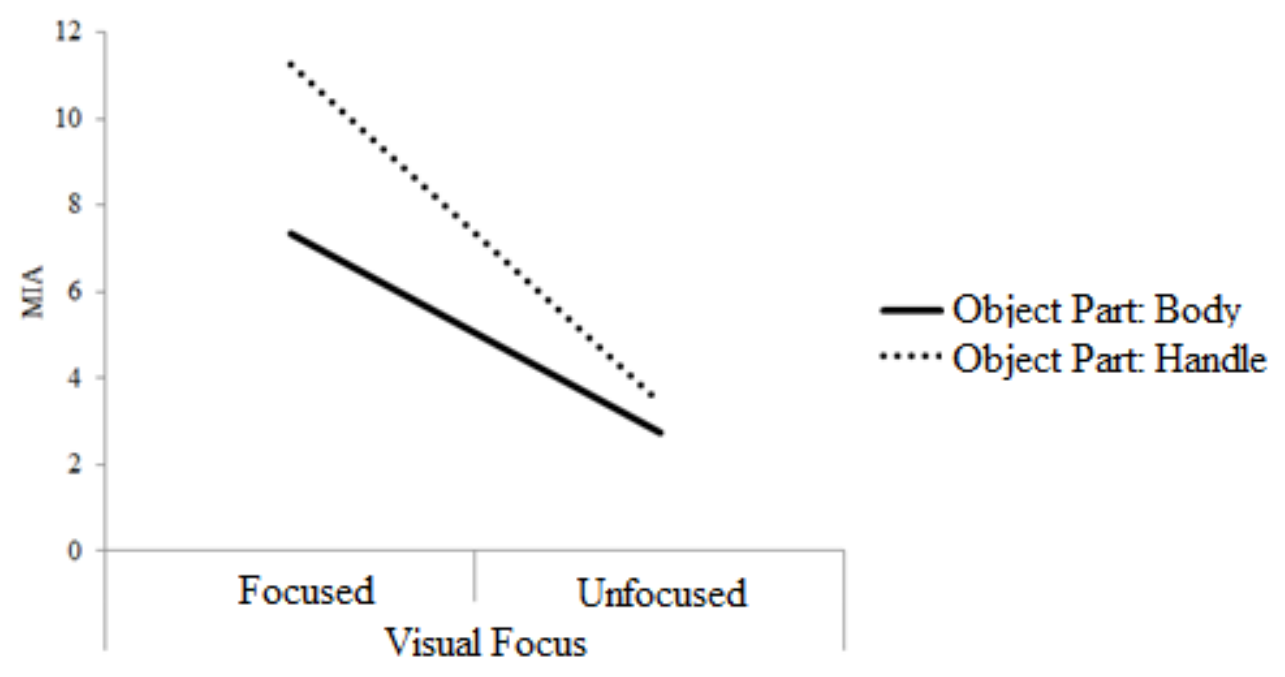

Figure 5. Experiment 1. Proportional Total Dwell Times. Visual Focus x Object Part. 


\section{Discussion}

The results of Experiment 1 confirmed a number of hypotheses and provided new important evidence. First, viewers were faster to identify visually focused targets. Second, a distractor's presence resulted in slowed target identification when the distractor object's handle was congruent with the responding hand. These findings are consistent with previous reports (e.g., Ellis, Tucker, Symes, \& Vainio, 2007) about the inhibitory nature of the distractor congruency effect on the identification of co-present manipulable targets. Third, although the main effect of target congruency did not reach significance in the manual RT data, its influence was revealed in the reliable interaction between Target Congruency and Visual Focus. As predicted, viewers identified response-congruent targets faster when they appeared in the focus of the viewer's visual attention. Presenting distractors in visual focus and targets outside of it (here, in the foreground) resulted in a negative compatibility effect for the target. Together, these findings provide evidence that attribution of affordance effects is subject to enhancement by means of the object's attentional status and that spatially guided visual attention activates relatively rich object representations that encode the object's action properties. Finaly, our data demonstrate that directing spatial attention to an object results in inhibition of actions associated with targets outside of the fixated region. (cf. Symes et al., 2010).

Our eye-tracking analysis provided novel evidence about the role of the objects' manipulable parts in potentiating affordance effects. Whilst identifying manipulable objects, viewers spent proportionally more time looking at the objects' handles than their bodies. This main effect of Object Part was accompanied by an important interaction with Object Focus: Viewers' attention for handles was further enhanced when the object was in visual focus. Together these results demonstrate that graspable objects' functional parts (i.e., their handles) automatically attract attention even when the experimental task is unrelated to manually manipulating these objects. Moreover, visually focusing the object 
further enhanced the handle-specific attention attraction effects. This novel finding provides initial support to the hypothesis outlined in Borghi (2012) - that visual cues to manipulable objects modulate variable affordances, such as the processing of handle orientation. By the same account linguistic cues (e.g., names or verbs) do not have this capacity. This should be true because linguistic information (e.g., the word cup) activates off-line or stable representational components, such as weight, general shape, and the general presence/availability of canonical graspable parts(i.e., handles), but not their variable orientations as permanently storing such transient features is presumed to be inefficient. This part of Borghi's claim was tested in Experiment 2.

\section{Experiment 2}

In order to independently test the ability of linguistic and visual cues to affect the attribution of grasp affordance effects, we added a name cue manipulation in Experiment 2 to the visual cueing manipulation already used in Experiment 1.

\section{Design}

The implemented design was similar to Experiment 1 with the addition of the new linguistic cueing factor "Name Focus". Manipulating Target Congruency (Congruent/Incongruent), Distractor Congruency (Congruent/Incongruent), Visual Focus (Target/Distractor), and Name Focus (Target/Distractor) resulted in a $2 \times 2 \times 2 \times 2$ withinsubjects design. Name Focus manipulation was operationalized via participants hearing the name of either the target or the distractor object during the presentation of the blank screen preceding the onset of the target display.

All names unambiguously related to one of the two objects. The names were singular nouns naming the object (i.e., cup, pan, saucer, strainer, brush, chisel, saw, and screwdriver). Otherwise, the analysis logic, the temporal interest period, and the interest areas for the eye-tracking analysis were the same as in Experiment 1.

\section{Materials}


We used the same visual displays as in Experiment 1. The eight object names were recorded by a male native speaker of English. The files' length was not controlled as the corresponding names differed in their syllabic structure. However, this did not present analysis-related problems as the names were always played within the 1000-ms blank screen event preceding object onset. Similarly to Experiment 1, a random selection of 16 experimental trials was used for practice.

\section{Apparatus}

The same apparatus as in Experiment 1 was used. The auditory stimuli were presented to the participants via Sennheiser ${ }^{\circ}$ headphones.

\section{Participants}

Twenty native speakers of English (8 males) at Dundee University with normal or corrected-to-normal vision participated for credit or $£ 6$. Their mean age was 21.2 years and their average handedness score was 34.9 (range 29-36), confirming they were all righthanded.

\section{Procedure}

The same procedure as in Experiment 1 was followed, with the exception that an unpredictive object name was played during the blank screen interval preceding the target display. A typical experimental sequence is portrayed in Figure 6.

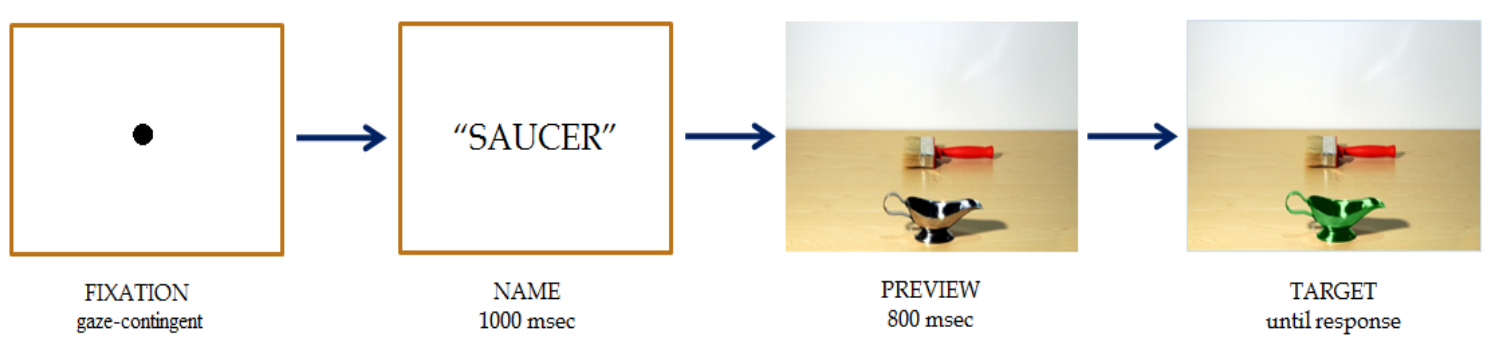

Figure 6. Experimental trial sequence in Experiment 2.

\section{Results}

Data from two participants were excluded from analysis due to high error rates (over $15 \%$ of their total responses). The data from the remaining 18 participants were 
subjected to statistical analyses. There was again no effect of response to category mapping on any dependent variables; therefore we aggregated across this factor.

\section{Manual reaction time analysis}

The same data trimming as before left us with $94 \%$ of the correct RT data for statistical analysis. Average manual RT in Experiment 2 was 598 ms. A 2x2x2x2 ANOVA revealed a reliable effect of Name Focus $(F(1,17)=31.800, p<.001)$ with the named targets identified $41 \mathrm{~ms}$ faster $(M=578 \mathrm{~ms})$ than when the distractor was named $(M=619 \mathrm{msec}$. $)$. There were no other main effects on the manual RT performance. However, RT data analysis revealed a reliable two-way interaction between Visual Focus and Name Focus $(F(1,17)=5.722, p=.029)($ see Figure 7$)$.

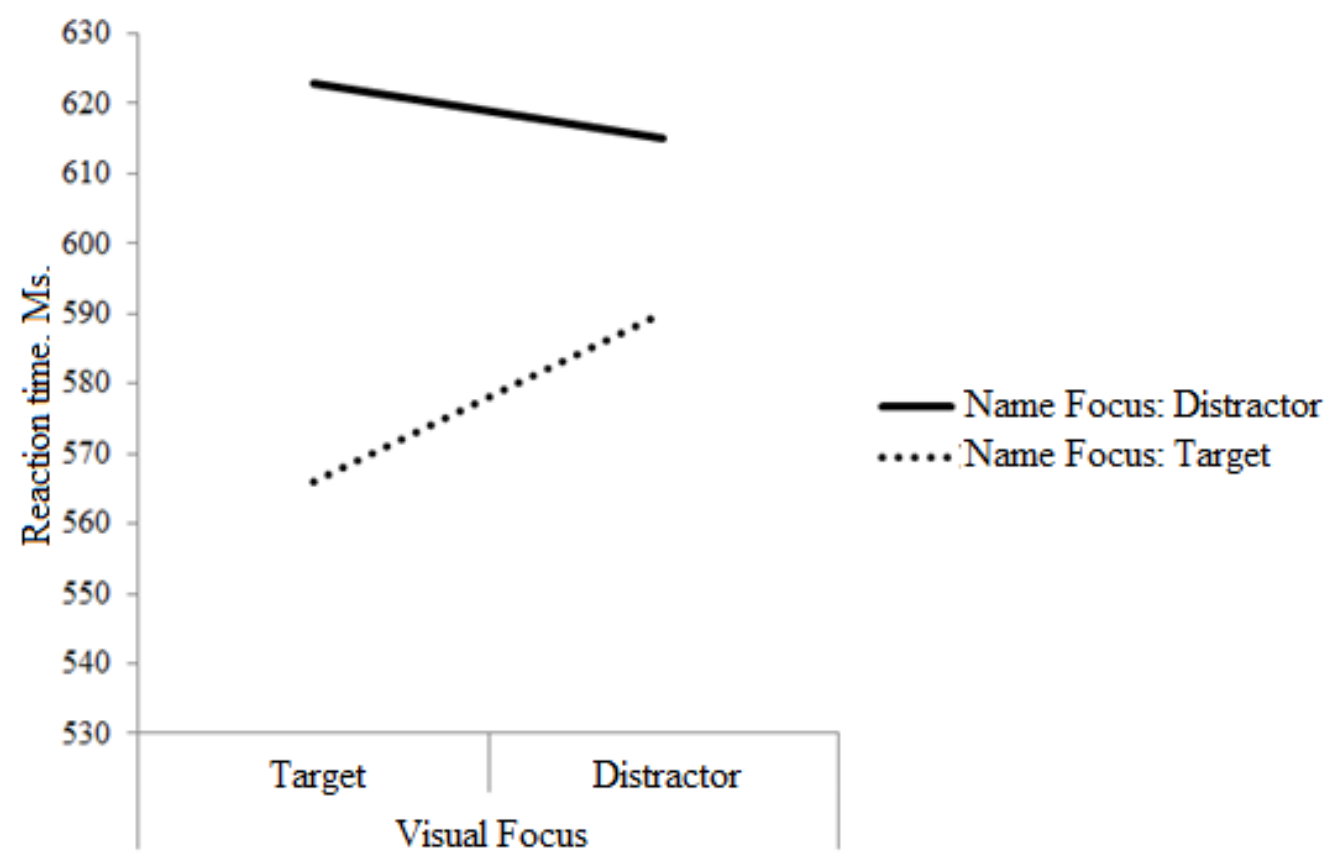

Figure 7. Experiment 2. Manual RTs for target detection. Visual Focus x Name Focus.

Planned comparisons confirmed that participants were $56 \mathrm{msec}$. faster to identify named and visually focused targets $(M=566 \mathrm{~ms})$ than named and focused distractors $(M=$ $622 \mathrm{~ms}), t(17)=5.487, p<.001$. The $26 \mathrm{msec}$. advantage for named and unfocused targets $(M=589 \mathrm{~ms})$ over named and unfocused distractors $(M=615 \mathrm{~ms})$ was also reliable $(t(17)$ $=3.208, p=.005)$. Manual RTs to targets were also $23 \mathrm{~ms}$ shorter when the named targets 
were unfocused than when they were focused $(t(17)=-2.722, p=.014)$. The $33 \mathrm{~ms}$ facilitation in the target unfocused/target named condition as compared to the target focused/distractor named condition was also reliable $(t(17)=4.001, p=.001)$. Overall, the interaction pattern demonstrates a combined effect from linguistic (naming) and visual (positioning) cueing of targets. Conversely, when the name participants received before the target display referred to the subsequent focused distractor this cueing combination resulted in a combined inhibition effect. The observed interaction between linguistic and visual cueing effects provides evidence about the presence of a combined effect on target identification from top-down linguistic and bottom-up visual cues.

We also registered two important reliable three-way interactions. One of such interactions was between Visual Focus, Target Congruency, and Distractor Congruency $(F(1,17)=8.164, p=.011)$. In order to graphically illustrate this interaction, we separated the RT data into two two-way interactions along the Visual Focus variable (target/distractor) (see Figures 8 and 9).

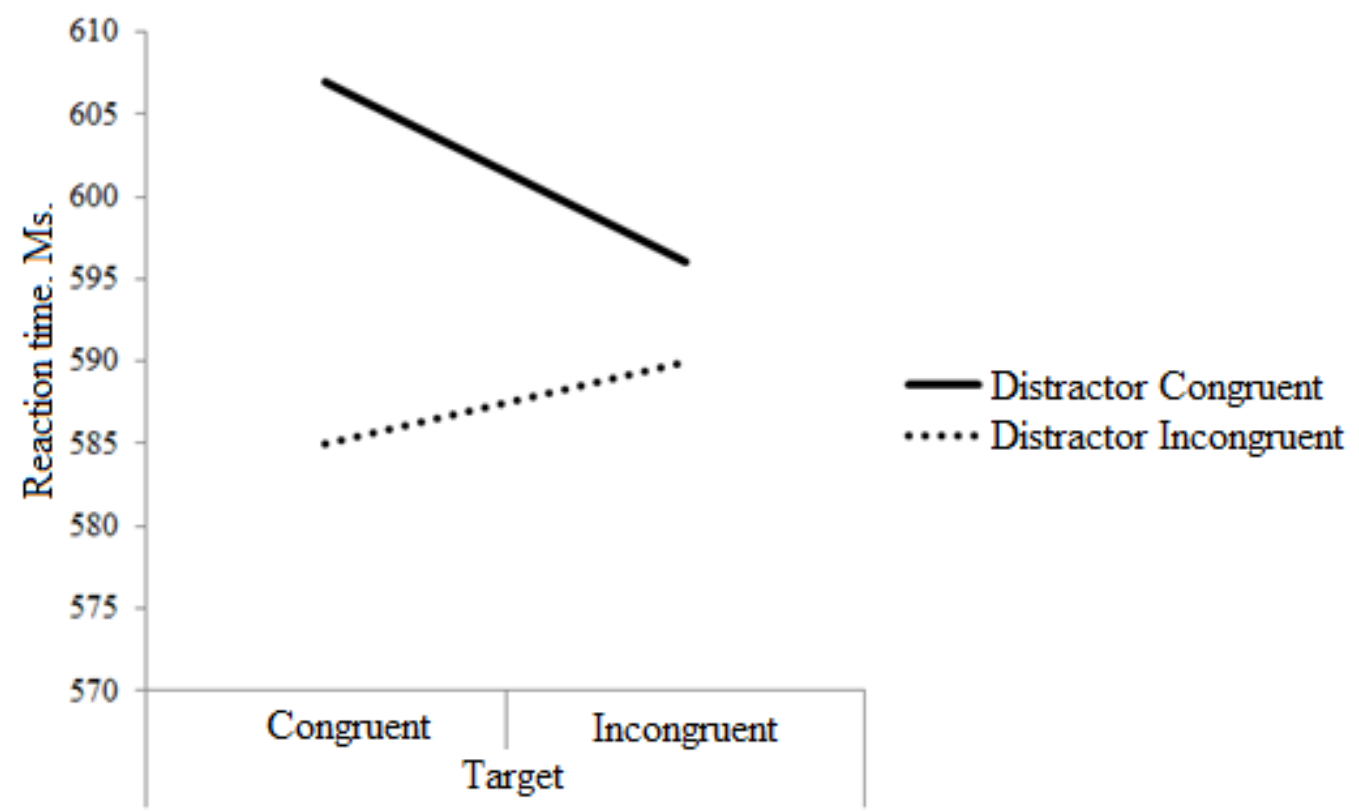

Figure

8. Experiment 2. Manual RT for Target detection in "Visual Focus Target" condition. 


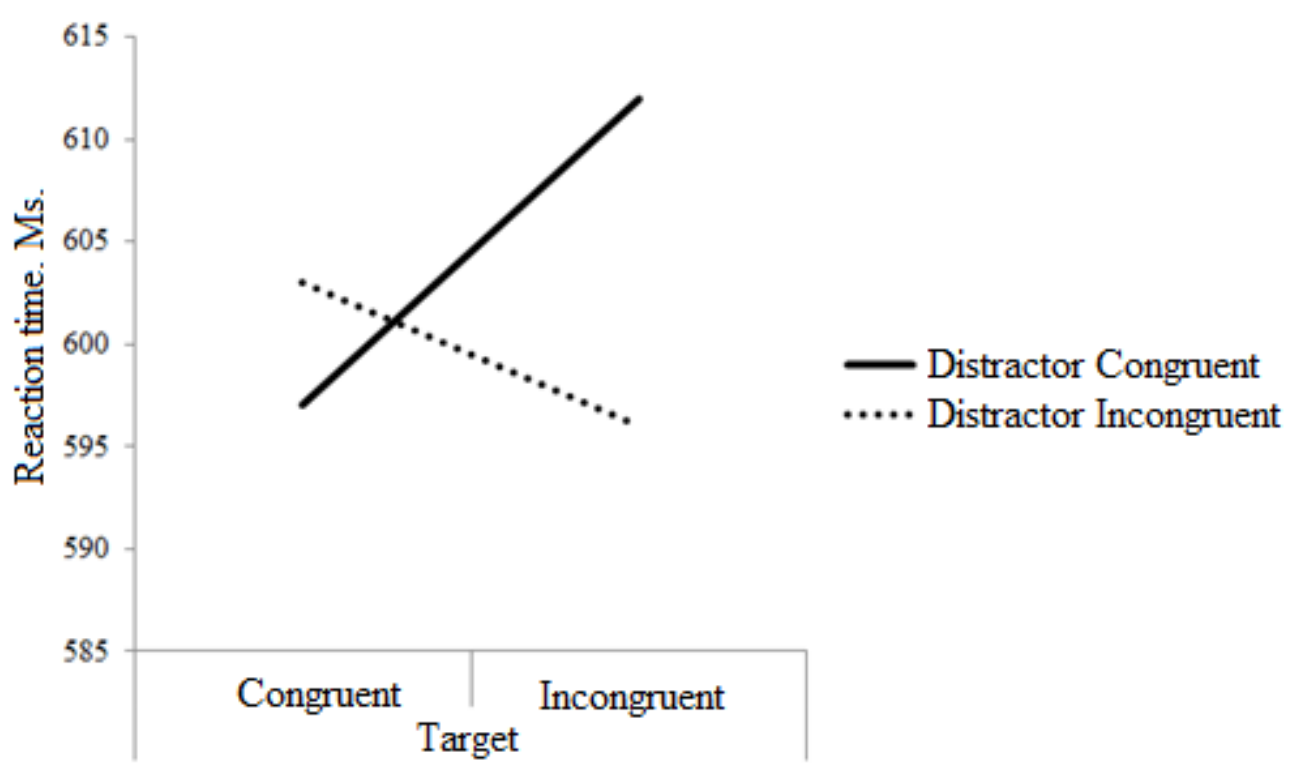

Figure 9.

Experiment 2. Manual RT for Target detection in "Visual Focus Distractor" condition.

When the target was visually focused (Figure 8), participants were $21 \mathrm{~ms}$. faster to identify congruent targets co-presented with incongruent distractors $(M=585 \mathrm{~ms})$ compared to the average of $606 \mathrm{~ms}$ when both objects were congruent $(t(17)=2.032, p=$ .058). When the distractor was visually focused (Figure 9), participants were faster to identify incongruent targets alongside incongruent distractors $(M=596 \mathrm{~ms})$ than in the situation when incongruent targets appeared alongside congruent distractors $(M=611 \mathrm{~ms})$ $(t(17)=1.917, p=.072)$. The general interaction pattern suggests the presence of negative compatibility effect when the target is in focus and positive compatibility effect when the distractor is in focus. We will provide a full examination of this novel finding in General Discussion.

Another reliable three-way interaction observed in manual RT data was between the factors of Target Congruency, Visual Focus, and Name Focus $(F(1,17)=7.490, p=$ $.014)$. Figures 10 and 11 illustrate this interaction along the Target Congruency factor. 


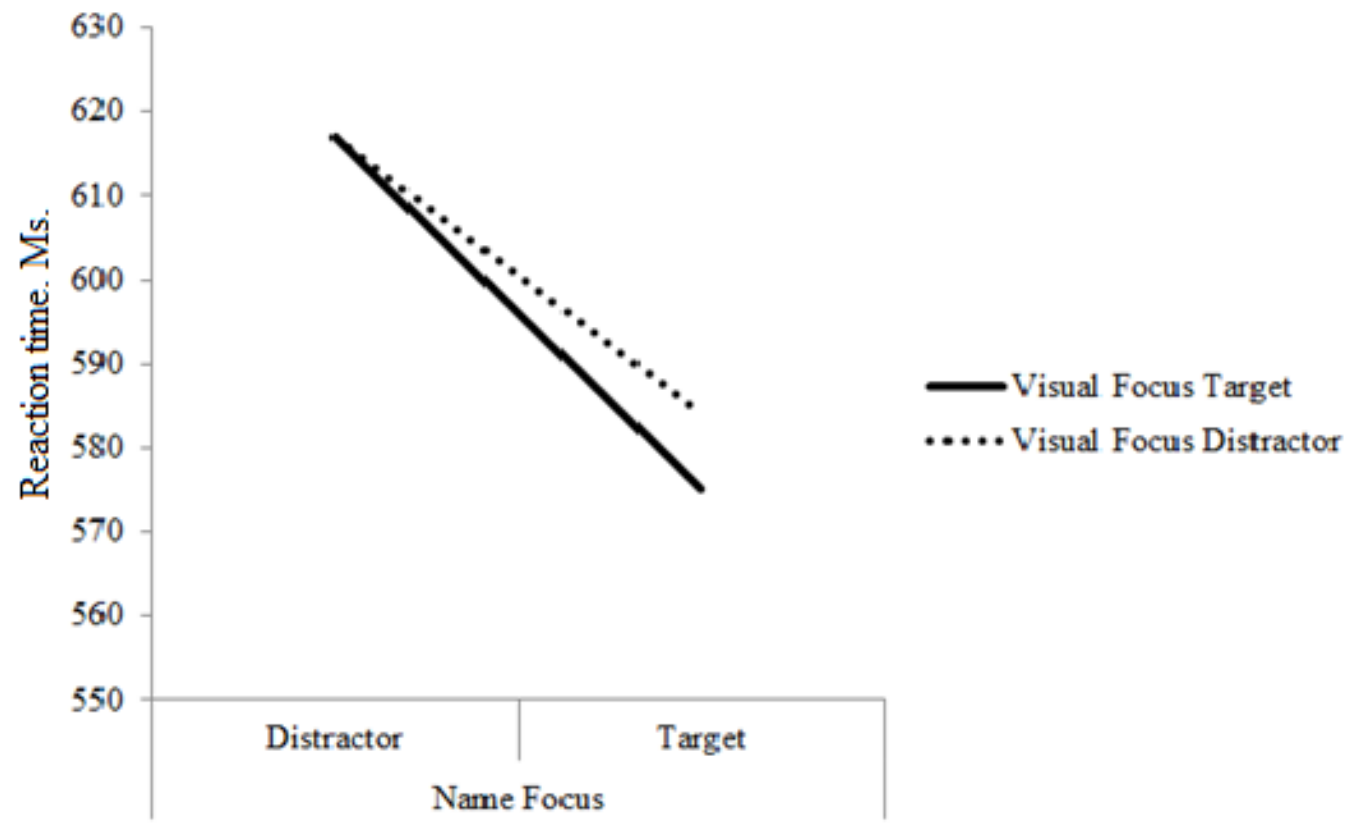

Figure 10. Experiment 2. Manual RTs for Congruent Targets.

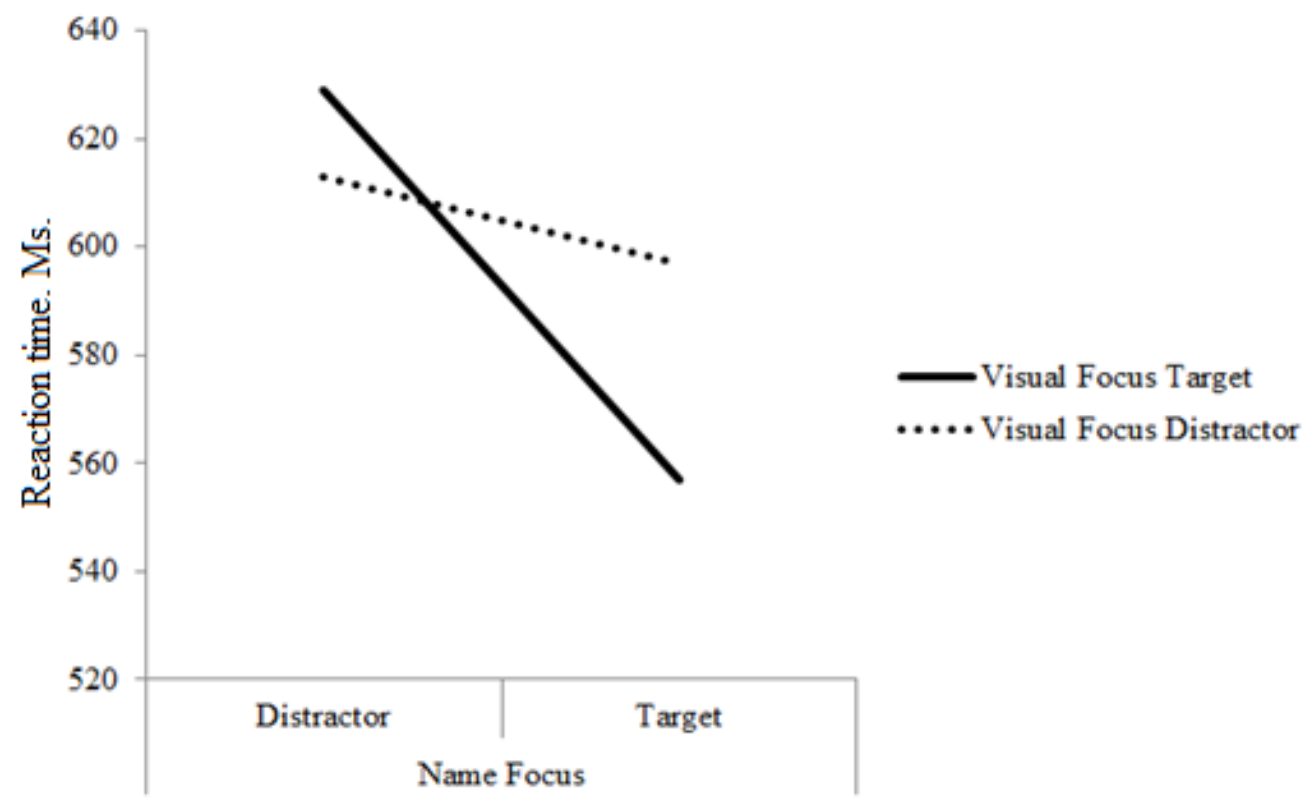

Figure 11. Experiment 2. Manual RTs for Incongruent Targets.

Figure 10 illustrates the RT pattern for trials when targets were presented with the handle orientation congruent with manual response. The pattern is carried primarily by the main effect of Name Cue with named congruent targets identified faster than when the distractor was named. It also shows an additional facilitation for congruent targets when they were simultaneously visually and linguistically focused. Hence, in case of target 
congruency, we observe a combined attentional facilitation by means of simultaneously providing top-down linguistic and bottom-up visual (positional) cues.

When the target appeared response-incongruent (Figure 11), a partially similar pattern was observed: Incongruent targets also enjoyed a combined facilitating effect from focusing via linguistic and visual cues but only when the distractor was out of visual focus. Putting distractor in the visually focused position slowed down target identification.

\section{Eye-tracking analysis.}

Our eye-tracking analysis of the proportional dwell times followed the same logic already discussed for Experiment 1 . However, we now also added a new factor, Name Focus. So, we implemented a 4-way design with the following factors: Visual Focus (Focused/Unfocused) X Name Focus (Focused/Unfocused) X Object Status (Target/Distractor) X Object Part (Body/Handle). A 4-way ANOVA performed on revealed reliable main effects of all four independent factors: Visual Focus $(F(1,17)=$ $136.541, p<.001)$, Name Focus $(F(1,17)=7.899, p=.012)$, Object Status $(F(1,17)=$ 21.123, $p<.001)$, and Object Part $(F(1,17)=168.423, p<.001)$. All effects were in the same direction as in Experiment 1: Participants looked more at visually focused than unfocused objects ( $M s=10$ MIA and 4 MIA, respectively), they looked more at named objects than not-named ( $M s=7$ MIA and 6 MIA, respectively), they looked more at targets than distractors ( $M S=7$ MIA and 6 MIA, respectively), and they looked more at handles ( $M=8$ MIA) than at bodies ( $M=5$ MIA). Hence, our data from Experiment 2 largely replicated the findings from Experiment 1 and also provided new evidence, namely, that linguistic cues to objects also implicitly attract viewer's attention.

Importantly, we also replicated the interaction between Visual Focus and Object Part registered in Experiment $1(F(1,17)=31.846, p<.001)$ with the same interaction pattern (see Figure 12). 


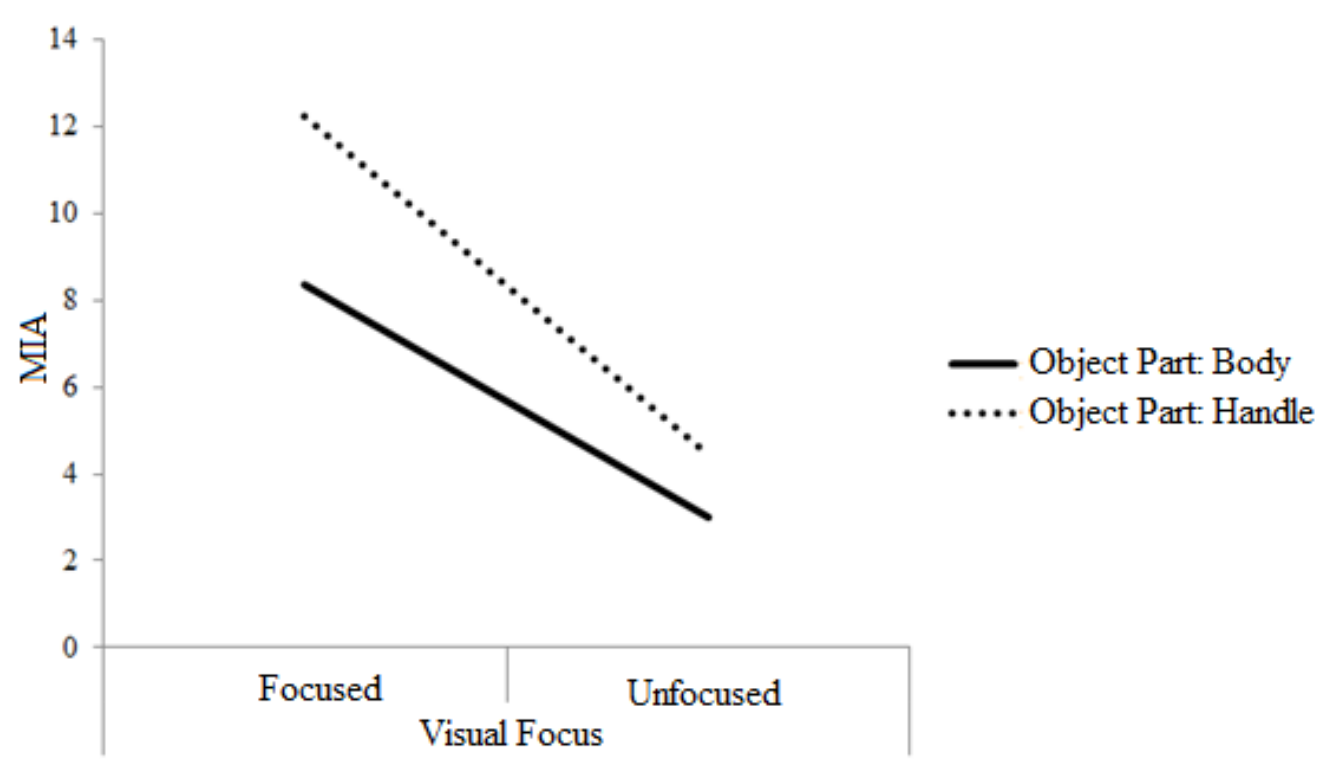

Figure

12. Experiment 2. Proportional Dwell Times. Object Position x Object Part.

Once again, handles attracted proportionally longer gazes than bodies, more so, when the object was in the focus of the viewer's visual attention. However, the interaction between Name Focus and Object part was unreliable $(p=.75)$.

\section{Discussion}

In Experiment 2, we introduced an additional variable to the design previously implemented in Experiment 1 - the name of either the Target or the Distractor prior to the presentation of the Target display. We aimed to investigate activation of grasp affordance effects in a mixed-cue scenario where both the top-down linguistic cues and the bottom-up visual cues are available to the perceiver. Our analysis confirmed that providing an object's name generally facilitated identification and that combining name cueing with visual cueing further accelerated object recognition. Our primary interest, however, was in understanding how these cues together affect the extent of affordance effects.

The three-way interaction between target and distractor congruency effects, on one hand, and the visual focus effect, on the other, revealed a complex pattern. Identification of the visually focused targets was faster when they were presented alongside an incongruent distractor. This facilitation effect reinforces previous findings about the negative 
compatibility effect from congruent distractors on graspable target identification (Ellis, et al. (2007). However, when the visually focused object was the distractor instead of the typical negative compatibility effect from co-present distractors we observed a positive compatibility effect: Viewers' benefitted in cases when targets and distractor had the same handle orientation; when objects were differently oriented target identification was slowed down. We will return to the theoretical analysis of this novel finding in General Discussion.

Name focus interacted with visual focus and the target congruency effect. For congruent targets, there was a clear combined facilitation effect from both cues with a slight additional benefit for visually focused targets over the unfocused ones. In case of incongruent targets, a partially similar pattern was observed: Named and visually focused targets were processed faster. However, putting the distractor in the visual focus resulted in an interference effect. This suggests that integration of linguistic and visual cues during object identification is limited to the objects that need to be identified, that is, target objects. When different cues direct attention to the co-present distractors, such an effect is not observed.

Finally, Experiment 2 confirmed our initial finding about the special role of handles as canonical graspable object parts in the establishment of the affordance effects. It also provided new evidence about differential effects of visual and linguistic cues on implicit attraction of attention by graspable parts. We replicated our findings from Experiment 1 by showing that activation of grasp-related affordances is based on implicit preferential attention to the object's handles and that this tendency is amplified by directing visual attention to the object. The same was not true in the case of linguistic cues (i.e., object names) as we failed to register either a three-way interaction between the two cueing manipulations and the Object Part or a two-way interaction between Name Cue and Object part. This is important as it provides initial empirical support to the hypothesis offered in 
Borghi (2012) - that visual but not linguistic cues have the potential to modulate variable affordances, such as compatibility effects obtained from congruent vs. incongruent handle orientations.

\section{General Discussion}

In two experiments, we analysed how perceptual and linguistic cues to the manipulable objects influence manual and oculomotor behaviour during identification of graspable objects. Object identification in a real-world scenario typically involves the availability of both visual and linguistic information about the objects. Hence, it is important to understand how the perceptual and the linguistic information are simultaneously integrated in simulating action components of manipulable objects during their identification.

One important general finding in both Experiments was that, albeit traced in different main effects and interactions, both target-bound and distractor-bound responsecongruency effects were registered in the manual reaction time data and in the eye-tracking data likewise. First, our analysis of the manual reaction-time data partially replicated the previously reported opposite-direction effects from target congruency and distractor congruency; that is, that response-congruent orientation of the target object's manipulable part generally facilitates the identification of this object while the response-congruent orientation of the manipulable part of a co-present distractor leads to the establishment of an inhibition effect on the speed of this identification.

However, our main focus was in understanding of how the availability of different types of cues to the objects - visual and linguistic - affects attribution of the affordance affect, generally and in relation to specific response-congruency factors. Both types of cues were previously shown to activate and amplify activated affordance effects. The novelty of our investigation was two-fold. First, there is no comparable analysis of the combined effect the top-down and the bottom-up cues exert on affordance effects in general and, 
specifically, the orientation compatibility effects. Second, our novel approach to analysing viewers' oculomotor behaviour documented a fine-grained record of implicit attentional processes underlying activation of affordance effects, especially in relation to the two implemented cueing manipulations.

In Experiment 1, we documented a general visual focusing facilitatory effect on object recognition both in manual reaction time data and in eye behaviour. Importantly, we were able to confirm the putting the object in the visual focus amplifies the observed congruency effect. This was revealed in the interaction between Visual Focus and Target Congruency in Experiment 1: Participants were faster to identify targets when their orientation was response-congruent and when they were in the attentional focus.

We also traced the visual focus effect in both target and distractor congruency profiles in the manual RT data in Experiment 2. A corresponding three-way interaction confirmed that when the target was focused its identification was faster if the target's orientation was response-congruent while the distractor's orientation was responseincongruent. This finding further extends previous research by showing that when target is in focus attentional resources are necessary to inhibit the congruency effect from copresent distractors as attentional facilitation of target detection can only be observed when the processor does not have to perform this inhibition operation; that is, in the case of incongruent co-present distractor.

When the distractor was focused, the pattern was reversed: Viewers were almost equally fast to detect congruent targets alongside congruent distractors and incongruent targets alongside incongruent distractors. Hence, instead of inhibition from simultaneously congruent or simultaneously incongruent distractors, target identification was facilitated in both cases. This new finding provides initial evidence about the existence of a differential attentional mechanism for the combined effect of the target- and distractor-related affordances in cases when targets or distractors are in visual focus. We suggest that in 
order to understand this complex pattern one needs to consider the identity of the object (target vs distractor) in the visual focus and the associated necessity to switch attention in the cases when this object is a distractor.

Imagine the case when the focused object is the target. The viewer's attention is already on this object before its target status is revealed. So, the observer temporarily considers both objects as potential targets while waiting for the colour change. Although attentional resources are "stretched" between the two objects, the primary focus is maintained on the fixated object; when it becomes the target. In this case, once the target identity is confirmed for the focused object, the information about the other object (i.e., the distractor) needs to be inhibited similarly to the IOR effect (Posner \& Cohen, 1984) or a negative priming effect (Neumann \& DeSchepper, 1992; Tipper, et al., 1991).

Having a distractor in the visual focus initially follows the same scenario. However, when the target is revealed attention needs to be switched from the focused distractor toward the unfocused target. In this case shared handle direction between distractor and target facilitates identification. This happens because the distractor's identity can be discarded when the observer realizes that the focused object is not the target. As a result, the distractor's identity does not need to be actively inhibited, leaving resources to accommodate both congruency profiles in a "boost" or "priming" fashion. This is a novel finding that suggests that affordance effects are activated differently in the situations when attention needs to be directed away from distractor objects in order to identify target objects.

Participants in Experiment 2 had two cues simultaneously available to them: Both objects could be linguistically and visually cued before one them appeared as the target. Unsurprisingly, the availability of the target's name proved to have had a very strong individual priming effect on object identification. Also, name and visual cues interacted in facilitating target identification process. 
Naturally, we were more interested in how simultaneously available perceptual and linguistic cues affect utilization of affordance parameters, such as target and distractor response congruency orientations. Two notable interactions reflected a degree of this influence. First, a reliable three-way interaction between Name, Target Position, and Target Congruency revealed combined effect of the two cue types in facilitating congruent target detection. Response-incongruent target were also processed faster when they were named but only when the distractor was out of visual focus. Putting distractor in the visually focused position slowed down target identification. This novel evidence about the effect of attentional facilitation on the objects' response congruency puts specific constraints on the degree and the scope of interactions between competing cues that can potentially lead to the attribution of affordance effects.

Finally, we used a very novel type of eye-tracking data analysis in both experiments. We analysed proportional viewing dwell times on targets and distractors with specific focus on the proportional dwell times spent on the nonmanipulable parts of the objects (e.g., bodies) and the manipulable parts of the objects (e.g., handles). Our motivation was that in order to utilize affordance profiles projected by the visual object, viewers may have to pay specific attention to its manipulable part. Our analysis confirmed this expectation: Participants visually interrogated handles as affordance-related object parts more than the objects' bodies. This handle-specific attention attraction was accompanied with a reliable interaction with Visual but not with Name cue manipulation: Activation of grasp-related affordances (based on implicit preferential attention to handles) was amplified in both studies by directing visual attention to the perceived object; the same amplification was not observed in the Name Cue condition. This new evidence reveals for the first time differential effects of visual and linguistic cues on implicit attraction of attention by graspable parts. This finding is important as it confirms the idea that top-down linguistic information activates offline (stable) memory object representations and that 
these representations do not encode the object's temporary or variable parameters, such as handle orientations. Bottom-up visual cues, on the other hand, have a potential to modulate variable affordances, such as compatibility effects obtained from handle orientations (Borghi, 2012). ${ }^{1}$

Together, our data cast light the complex perceptual and linguistic attentional mechanisms underlying the activation of grasp-related affordance effects. One important further question is whether attention in this case is always deployed overtly? Affordance effects tend to be relatively small; hence, it is natural to expect that at least in part, the attentional mechanism can be covert rather than overt. Further experiments will address this issue.

\footnotetext{
${ }^{1}$ Of course, upon hearing cup one would not only represent its typical shape and weight but also assume that a typical cup has a handle. However, what would not be available from hearing cup is how this handle is oriented because the semantic information in cup does not cue a particular handle orientation and/or location. A richer linguistic cue (e.g., cup with a handle on the left) should, in principle, activate both stable and variable affordances.
} 


\section{References}

Adamo M. \& Ferber S. (2009). A picture says more than a thousand words: Behavioural and ERP evidence for attentional enhancements due to action affordances. Neuropsychologia, 47, 1600-1608.

Ambrosini, E., Scorolli, C., Borghi, A.M., Costantini, M. (2012). Which body for embodied cognition? Affordance and language within actual and perceived reaching space. Consciousness and Cognition, 21, 1551-1557.

Anderson, S.J., Yamagishi, N., Karavia, V. (2002) Attentional processes link perception and action. Proceedings of the Royal Society, Series B, 269, 1225-1232.

Barsalou, L.W. (2008). Grounded Cognition. Annual Review of Psychology, 59, 617-645.

Borghi, A.M. (2012). Action language comprehension, affordances and goals. In Y. Coello, A. Bartolo (Eds). Language and action in cognitive neuroscience. (pp. 531-556). Psychology Press.

Borghi, A.M. \& Riggio L. (2009). Sentence comprehension and simulation of object temporary, canonical and stable affordances. Brain Research, 1253, 117-128.

Bub, D.N., Masson, M.E.J., \& Cree, G.S. (2008). Evocation of functional and volumetric gestural knowledge by objects and words. Cognition, 106, 27-58.

Bub, D.N., \& Masson, M.E.J. (2010). Grasping beer mugs: On the dynamics of alignment effects induced by handled objects. Journal of Experimental Psychology: Human Perception and Performance, 36, 341-358.

Costantini, M., Ambrosini, E., Scorolli, C., Borghi, A.M. (2011).When objects are close to me: affordances in the peripersonal space. Psychonomic Bulletin \& Review, 18, $32-38$.

Craighero, L., Fadiga, L., Rizzolatti, G. \& Umiltà, C. (1999). Action for perception: a motor-visual attentional effect. Journal of Experimental Psychology: Human Perception and Performance. 25, 1673-1692. 
Craighero, L., Bello, A., Fadiga, L., \& Rizzolatti, G. (2002). Hand action preparation influences the responses to hand pictures. Neuropsychologia, 40, 492-502.

Creem, S.H., \& Proffitt, D.R. (2001). Grasping objects by their handles: A necessary interaction between cognition and action. Journal of Experimental Psychology: Human Perception and Performance, 27, 218-228.

Derbyshire, N., Ellis, R., Tucker, M. (2006). The potentiation of two components of the reach-to-grasp action during object categorisation in visual memory. Acta Psychologica 122(1), 74-98.

di Pellegrino, G.; Rafal, R.; \& Tipper, S.P. (2005). Implicitly evoked actions modulate visual selection: Evidence from parietal extinction. Current Biology, 15(16), $1469-1472$.

Ellis, R. \& Tucker, M. (2000). Micro-affordance: The potentiation of actions by seen objects. British Journal of Psychology, 91, 451-471.

Ellis, R., Tucker, M., Symes, E., \& Vainio, L. (2007). Does selecting one visual object from several require inhibition of the actions associated with non-selected objects? Journal of Experimental Psychology: Human Perception and Performance, 33, 670-691.

Fischer, M. H., \& Dahl, C. (2007). The time course of visuo-motor affordances. Experimental Brain Research, 176(3), 519-524.

Gibson, J.J. (1979). The Ecological Approach to Visual Perception. Boston: Houghton Mifflin.

Goodale, M.A. (2011). Transforming vision into action. Vision Research, 51, 1567-1587. Humphreys, G.W., Yoon, E. Y., Kumar, S., Lestou, V., Kitadono, K., Roberts, K.L., \& Riddoch, M.J. (2010). The interaction of attention and action: From seeing action to acting on perception. British Journal of Psychology, 101, 185-206. 
Klatzky, R.L., Pellegrino, J.W., McCloskey, B.P., \& Doherty, S. (1989). Can you squeeze a tomato? The role of motor representations in semantic sensibility judgments. Journal of Memory and Language, 28, 56-77.

Lindemann, O., Stenneken, P., van Schie, H., \& Bekkering, H. (2006). Semantic activation in action planning. Journal of Experimental Psychology: Human Perception and Performance 32(3): 633-643.

Loach, D., Frischen, A., Bruce, N. \& Tsotsos, J.K. (2008). An attentional mechanism for selecting appropriate actions afforded by graspable objects. Psychological Science, 19, 1253-1257.

Martin, A. (2008). The representation of object concepts in the brain. Annual Review of Psychology, 58, 25-45.

Masson, M.E.J., Bub, D.N., \& Newton-Taylor, M. (2008). Language-based access to gestural components of conceptual knowledge. Quarterly Journal of Experimental Psychology, 71, 869-882.

Masson, M.E.J., Bub, D.N., \& Warren, C.M. (2008). Kicking calculators: Contribution of embodied representations to sentence comprehension. Journal of Memory and Language, 59, 256-265.

Michaels, C.F. (1989). S-R compatibilities depend on eccentricity of responding hand. Quarterly Journal of Experimental Psychology, 41(2), 263-272.

Michaels, C. F. (1993). Destination compatibility, affordances, and coding rules - a reply. Journal of Experimental Psychology-Human Perception and Performance, 19(5), $1121-1127$.

Neumann, E. \& DeSchepper, B.G. (1992). An inhibition-based fan effect: Evidence for an active suppression mechanism in selective attention. Canadian Journal of Psychology, 46, 1-40. 
Pappas, Z. \& Mack, A. (2008). Potentiation of action by undetected affordant objects. Visual Cognition, 16(7): 892-915.

Phillips, J.C. \& Ward, R. (2002). S-r correspondence effects of irrelevant visual affordance: Time course and specificity of response activation. Visual Cognition, $9(4-5), 540-558$.

Posner, M. I., \& Cohen, Y. (1984). Components of visual orienting. In H. Bouma \& D. G. Bowhui (Eds.). Attention and Performance (pp. 531-556), Vol. X., Hillsdale, NJ: Erlbaum.

Riggio, C., Iani, E., Gherri, F., Benatti, S., Rubichi, R., \& Nicoletti, R. (2008). The role of attention in the occurrence of the affordance effect. Acta Psychology, 127, 449458.

Schuch, S., Bayliss, A.P., Klein, C. \& Tipper, S.P. (2010). Attention modulates motor system activation during action observation: Evidence for inhibitory rebound. Experimental Brain Research, 205, 235-249.

Symes, E., Tucker, M., Ellis, R., Vainio, L., \& Ottoboni, G. (2008). Grasp preparation improves change detection for congruent objects. Journal of Experimental Psychology: Human Perception and Performance 34(4), 854-871.

Symes, E., Ottoboni, G., Tucker, M., Ellis, R., \& Tessari, A. (2009). When motor attention improves selective attention: the dissociating role of saliency. Quarterly Journal of Experimental Psychology 63 (7), 1387-1397

Tipper, S. P., Weaver, B., Cameron, S., Brehaut, J. C., \& Bastedo, J. (1991). Inhibitory mechanisms of attention in identification and localization tasks: Time course and disruption. Journal of Experimental Psychology. Learning Memory and Cognition, 17(4), 681-692. 
Tipper, S.P., Paul, M.A., \& Hayes, A.E. (2006). Vision for action: the effects of object property discrimination and action state on affordance compatibility effects. Psychonomic Bulletin and Review, 13(3), 493-498.

Tucker, M. \& Ellis, R. (1998). On the relations between seen objects and components of potential actions. Journal of Experimental Psychology: Human Perception and Performance, 24, 830-846.

Tucker, M. \& Ellis, R. (2001). The potentiation of grasp types during visual object categorization. Visual Cognition, 8, 769-800.

Tucker M. \& Ellis R. (2004). Action priming by briefly presented objects. Acta Psychologica, 116, 185-203.

Tschentscher, N., \& Fischer M. H. (2008). Grasp cueing and joint attention. Experimental Brain Research. Experimentelle Hirnforschung. Expérimentation cérébrale, $190(4), 493-498$.

Vainio, L., Ellis, R., \& Tucker, M. (2007). The role of visual attention in action priming. The Quarterly Journal of Experimental Psychology, 60, 241-261. 